\title{
N6-Methylandenosine-Related IncRNAs are Potential Biomarkers for Predicting the Overall Survival Rate of Patients Bladder Cancer
}

\section{Haihang Zhang}

Hainan Medical University https://orcid.org/0000-0002-5335-4502

\section{Panpan Xie}

Hainan Medical University

Kaijia Shi

Hainan Medical University

Danni Xu

Hainan Medical University

Xingrui Cai

Hainan Medical University

\section{Changcheng Yang}

Hainan Medical University

Jiangzheng Zeng

Hainan Medical University

Yanda Lu ( $\nabla$ yandalu986@163.com )

Hainan Medical University https://orcid.org/0000-0002-1386-6001

\section{Primary research}

Keywords: bladder cancer, N6-methylandenosine methylation, long non-coding RNA, immune microenvironment

Posted Date: June 2nd, 2021

DOI: https://doi.org/10.21203/rs.3.rs-534193/v1

License: (c) (i) This work is licensed under a Creative Commons Attribution 4.0 International License. Read Full License 


\section{Abstract}

Background: The prognostic value of N6-methylandenosine-related long non-coding RNAs (m6A-related IncRNAs) was investigated in 414 bladder cancer (BLCA) and 19 normal bladder tissue samples from The Cancer Genome Atlas (TCGA) datasets.

Methods: We implemented Pearson correlation analysis to explore the IncRNAs associated with m6A, and then performed univariate Cox regression analysis to identify nine m6A-associated IncRNAs with prognostic value. The patients with BLCA were divided into two subgroups by consistency clustering. Analysis of the two groups of immune cell infiltration, immune microenvironment and clinical results were significantly different. We identified the prognostic significance of m6A-related IncRNAs by bioinformatic and statistical analysis of data from patients with BLCA.

Results: We constructed an m6A-related IncRNA prognostic signature (m6A-LPS, including AL136295.2, AC104564.3, ATP1B3-AS1, EHMT2-AS1 and AC116914.2) to predict the OS of BLCA patients. It is proved that m6A LPS has independent prognostic value.

\section{Materials And Methods}

\section{Datasets And M6a-related Genes}

mRNA expression files [Fragments Per Kilobase of transcript per Million mapped reads (FPKM)normalized] and the corresponding clinicopathological data were acquired from the TCGA databases (https ://porta I. gdc. cance r. gov/). Ultimately, we obtained a involving 414 patients BLCA and 19 normal bladder tissue mRNA expression. After deleting samples with no statistical significance, 426 samples remained, including the data of cancer tissue $(n=407)$ and normal tissue $(n=19)$.

Simultaneously, we have obtained corresponding clinicopathological data from TCGA dataset. In addition, based on previous publications, expression matrixes of $21 \mathrm{m6A}$-related genes were extracted from the TCGA databases, respectively, including expression data on writers (METTL3, METTL16, WTAP, VIRMA, ZC3H13, RBM15 and RBM15B), erasers (FTO and ALKBH5) and readers (YTHDC1, YTHDC2, YTHDF1, YTHDF2, YTHDF3, HNRNPC, FMR1, LRPPRC, HNRNPA2B1, IGFBP1, IGFBP2 and RBMX including in molecular features)Table 1.

Table 1

The components of m6A RNA methylation regulators in writer-, reader- and eraser-complex.

\section{Regulators}

Writers METTL3, METTL16, WTAP, VIRMA, ZC3H13, RBM15 and RBM15B

Readers YTHDC1, YTHDC2, YTHDF1, YTHDF2, YTHDF3, HNRNPC, FMR1, LRPPRC, HNRNPA2B1, IGFBP1, IGFBP2 and RBMX

Erasers FTO and ALKBH5 


\section{Identification Of M6a-related Lncrnas}

We extracted IncRNA expression data from the mRNA expression data according to the GENCODE project (http://www.gencodegenes.org) [1, 2]. Next, Pearson's correlation analysis was performed between m6Arelated genes and IncRNA expression levels in samples to identify m6A-related IncRNAs according to the correlation coefficients and $p$ values $(\mid$ correlation coefficient $\mid>0.4$ and $p<0.001)$. To determine the prognostic value of m6A-related IncRNAs, we conducted univariate Cox regression analysis on the m6Arelated IncRNAs by using the "survival" package in $\mathrm{R}$ (v3.6.2), and the difference analysis between normal tissue and BLCA tissue, $p<0.05$ indicated that m6A-related IncRNAs were correlated with overall survival (OS) and considered prognostic m6A-related IncRNAs.

\section{Consensus Clustering For M6a-related Incrnas}

By consensus cluster analysis, we identified two different subgroups of BLCA individuals by the expression profile of m6A-related IncRNAs. The unsupervised clustering "Pam" method based on Euclidean and Ward's linkage was used in this analysis, executed by using the "ConsensuClusterPlus" $\mathrm{R}$ package [3].

\section{The Landscape Of Immunocell Infiltration In The Blca}

We used CIBERSORT to estimate the ratio of 22 infiltrating immune cell types based on each BLCA sample. CIBERSORT is an analytical algorithm, which can characterize cell composition of complex tissues based on normalized gene expression profiles [4]. ESTIMATE evaluated the immune and stromal contents (immune and stromal score) for each BLCA sample[5]. The results were tested by log-rank test. All the analyses were conducted using R programming language and Perl software.

\section{Bioinformatic Analysis}

Pearson correlation analysis was first implemented to mining m6A-related IncRNAs (with the | Pearson R| $>0.5$ and $p<0.001$ ) in each dataset for finding m6A-related IncRNAs. Then univariate Cox regression analysis was implemented to filtrate the prognostic m6A-related IncRNAs in the datasets. Use the "survival" package in R (v3.6.3) to verify the difference between the IncRNA of univariate Cox regression analysis between normal tissue and BLCA tissue. Thereafter, using the R package "glmnet" [6] to conduct least absolute shrinkage and selection operator (LASSO) Cox regression (with the penalty parameter estimated by 10-fold cross-validation)[7], we developed a m6A-related IncRNA prognostic signature for the BLCA patients involving 5 m6A-related IncRNAs. The risk score calculating formula is: 
Risk score $=\sum_{i=1}^{n} \operatorname{Coef}_{i} * x_{i}$

where Coef $_{i}$ means the coefficients, $x_{i}$ is the FPKM value of each m6A-related IncRNAs. Risk scores were computed for all patients including in two datasets. For both two datasets, m6A-Related IncRNAs in the high-risk subgroup in contrast to the low-risk subgroup were identified based on the standards of ( log2(Fold change) > 1 and $p<0.05$ ) using the R package “limma” [8]. Then the validity of the risk score was tested by survival analysis, risk plot and receiver operating characteristic (ROC) curve. Univariate and multivariate Cox regression analysis were performed to validate the independent role of the risk signature. Finally, the relevance of risk score and clinicopathological features, Immune Cell Infiltration were evaluated by $\mathrm{R}$ 3.6.3.

\section{Statistical Analysis}

The Kruskal-Wallis test was used to compare more than two groups, and the Wilcoxon test was used to compare two groups. The X-tile software, which iteratively tests possible cut points to select the one with the maximum rank statistic, was used to classify patients into two subtypes in each dataset and to reduce the computational batch effect. The Kaplan-Meier plotter was employed to generate survival curves for the subgroups in each dataset. The log rank test evaluated the statistically significant differences. Univariate and multivariate Cox regression analysis were utilized to evaluate the independent prognostic value of the m6A-LPS regarding OS [9]. The chi-square test analyzed the correlation between the subgroups of IncRNAs related to m6A and immune cell infiltration score, and the Spearman analysis computed the correlation coefficient. Two-tailed $p<0.05$ was considered as statistically significant.

\section{Introduction}

Bladder cancer (BLCA) is the most popular urologic cancer with distinctive morbidity and mortality throughout the world, and is the seventh most prevalent cancer in males. According to data of Global Cancer Statistics, about 440,000 new cases of BLCA are diagnosed while 130,000 patients died from it around the world every year [10]. In addition, BLCA has a high recurrence rate, and approximately half of patients relapse after radical surgery and present with metastases $[11,12]$. Platinum-based chemotherapy and new Immune Checkpoint Inhibitors has provided unprecedented benefits for patients with metastatic urothelial carcinoma, but the clinical effect of BLCA therapy is not significant [13]. To improve survival and reduce the burden of BLCA, researchers must develop novel biomarkers for better prediction of the prognosis and treatment response of BLCA.

m6A modification, the most abundant epigenetic methylated modification of mRNAs and non-coding RNAs, plays a vital role in RNA splicing, export, stability and translation [14]. m6A modification is a invertible and dynamical RNA epigenetic process that is regulated by m6A regulators, including "writers" 
(methyltransferases), "readers" (signal transducers) and "erasers" (demethylases) [15]. The effect of m6A modifications on IncRNAs may involve several regulatory mechanisms. On the one hand, m6A modifications might modulate the function of IncRNAs by providing a binding site for the m6A reader proteins or by modulating the structure of the local RNA to induce RNA-binding protein entry. On the other hand, m6A modifications might also regulate the relationship between IncRNAs and specific DNA sites by affecting the RNA-DNA triple helix structure [16]. Recent research had revealed that m6A modification can regulate oncogenesis and tumor progression in several kinds of cancers, including BLCA. For example, the long noncoding RNA X-inactive-specific transcript is proficient at gene-silencing transcription, which it performs by recruiting specific protein complexes to the $\mathrm{X}$ chromosome during female mammalian development [17]. In glioblastoma stem cells, an m6A demethylase, ALKBH5, was shown to interact with the IncRNA Forkhead box protein M1 (FOXM1)-AS to promote cancer cell proliferation and tumorigenicity. METTL3 is known to be involved in all stages in the life cycle of RNA. It affects the tumor formation by the regulation the m6A modification in the mRNAs of critical oncogenes or tumor suppressors. In BLCA, METTL3 could promote the BLCA progression via AFF4/NF-KB/MYC signaling network by a m6A dependent manner [18]. Thus, it would only be reasonable to attempt to integrate various methods, including dysregulation of m6A-Related IncRNAs regulators that have gathered enormous attention, to further improve of BLCA. However, the full role of m6A regulators in the dysregulation of IncRNAs in cancers remains unclear, and few studies have been performed to explore the mechanisms underlying how m6A modifications contribute to IncRNA-dependent BLCA occurrence and development. Thus, understanding how m6A modifications of IncRNAs are involved in BLCA progression may help to identify biomarkers that can act as useful therapeutic targets.

\section{Results}

\section{Identification Of M6a-related Lncrnas Involved In Blca Development}

Firstly, we identified 14086 IncRNAs in 407 BLCA samples from TCGA datasets. We then extracted the expression matrixes of $21 \mathrm{m6A}$-related genes from the TCGA datasets, respectively. A IncRNA which expression value was correlated with one or more of the 21 m6A-related genes (| Pearson $R \mid>0.4$ and $p<$ 0.001 ) was defined as a m6A-related IncRNAs. Pearson correlation analysis was performed to search m6A-related IncRNAs. we obtained 741 IncRNAs which were significantly correlated with m6A-related genes. The network diagram of co-expression between 741 IncRNAs and 21 m6A-related genes was shown in Fig. 1a. Combined with the prognostic information, univariate Cox regression was performed to screen the m6A-related IncRNAs related to the prognosis of from 741 IncRNAs related to m6A $(p<0.001)$. We found that $9 \mathrm{m6A}$-related IncRNAs were significantly correlated with the OS of BLCA patients (Fig. 1b). Then transcriptome profile of m6A-related IncRNA was thoroughly investigated. The information of IncRNA related to $\mathrm{m} 6 \mathrm{~A}$ was extracted, and the difference analysis was carried out between the normal tissues of bladder and BLCA tissues. The results showed that $9 \mathrm{~m} 6 \mathrm{~A}$-related IncRNAs had differences( ${ }^{\star} \mathrm{P}$ $<0.05,{ }^{* \star} P<0.01,{ }^{\star \star *} P<0.001$; Fig. 1C, d). 


\section{Correlation of the m6A-related IncRNAs with clinicopathological features of BLCA patients}

To develop a prognostic signature based upon 9 m6A-related IncRNAs, we sought to stratify 407 BLCA patient samples by consensus clustering analysis (Fig. 2a, b). Based on the cumulative distribution function (CDF) value, $\mathrm{k}=2$ was the optimal cluster number to divide the BLCA cohort, namely, cluster 1 and cluster 2 (Fig. 2C). Furthermore, we evaluated the associations between clusters and clinicopathological features. Compared with cluster 2, m6A-related IncRNAs were more highly expressed in cluster 1 (Fig. 2g). Moreover, we explored the prognostic implications of the IncRNA by integrating them with survival information. The analysis was performed by using the Kaplan-Meier plotter, and we found that patients in cluster 1 had a better prognosis, whereas patients in IncRNA clusters 2 had the unfavorable outcomes (log rank test, $p=0.016$; Fig. 2f). Studies have found that increased Foxp3 expression may be an independent predictor of poor prognosis of BLCA [19]. Targeted gene methylation modification provides new insights for clinical treatment design of some diseases. Understanding the characteristics and regulatory mechanism of Foxp3 protein is helpful to design a reasonable treatment plan for BLCA. Additionally, the two genomic clusters showed significant differences in Foxp3 expression levels. Compared with cluster 1, the expression level of Foxp3 in cluster 2 was relatively high (log rank test, $p<0.001$; Fig. 2d). In addition, Foxp3 expression in BLCA was higher than that in normal tissues (log rank test, $p<0.05$; Fig. 2e). At the same time, we analyzed the correlation between the m6A-related IncRNAs. Among them, we focused on the significant negative correlation between Foxp3 closely related to the PTOV1 - AS2, AC104564.3, EHMT2-AS1 and AC004148.1. (Fig. 2h). Taken together, the consistency between different IncRNAs clusters and prognostic profiles shows that our classification method is scientific and reasonable.

\section{Consensus clustering for m6A-related IncRNAs associated with distinct immune cell infiltration}

To unravel the underlying biological characteristics of distinct m6A-related IncRNAs subtypes. First, we performed the CIBERSORT algorithms to quantify the activity or enrichment levels of immune cells in BLCA tumor tissues. Among the two main m6A-Related IncRNAs subtypes, we performed differential analysis to determine the immune cell infiltration among these subtypes by limma packages of $R$ software. The cluster 1 exhibited the highest Tregs infiltration. Cluster 2 showed an escalated memory activated $C D 4^{+} T$ cells and immunosuppression associated $M 2$ macrophages and neutrophils infiltration (Fig. 3a). To investigate the effect of m6A related-IncRNA on the tumor immune microenvironment (TIME) of BLCA, we performed the ESTIMATE algorithms to quantify the activity or enrichment levels of immunoscore, StromalScore and ESTIMATE Score in BLCA tumor tissues. Compared with cluster 1, StromalScore,ESTIMATEScore and ImmuneScore are more highly expressed in cluster 2 (Fig. 3b-d). In addition, to elucidate the potential regulatory mechanisms resulting in differences in the m6A relatedIncRNA between the two subgroups, we performed gene set enrichment analysis (GSEA). The characteristics of the patient in cluster 1 were base excision repair, notch signaling pathway, mTOR signaling pathwayand and regulation of autophagy (Fig. 3e-h) and so on. Gene set enrichment analysis 
showed that there were several tumor and inflammatory markers enriched in cluster 2 subgroup, such as cytokine-cytokine-receptor-interaction, cell adhesion molecule cam, chemokine signal transduction pathway and T cell receptor signal transduction pathway (Fig. 3i-I). These results may give us some insights into the cellular biological effects associated with m6A-related IncRNA prognostic markers.

\section{Construction Of The M6a-lps In The Tcga- Train Dataset}

Firstly, sort out the clinical data of BLCA patients, and normal samples and samples without survival data were deleted. A total of $(n=407)$ patients were included for IncRNAs model construction. Using computergenerated random numbers, the entire TCGA-BLCA data set $(n=407)$ was divided into a TCGA-Train(204 cases) and a TCGA-Test (203 cases). The patient's survival time, survival status and m6A-related IncRNAs expression data were combined. Model construction in the TCGA-Train, and then the best model selection in the TCGA-Test. To build the the prognostic model of m6A-related IncRNAs for forecasting the OS of BLCA patients, we performed a LASSO Cox analysis on the basis of the 9 m6A-related prognostic IncRNAs in the TCGA-Train and it generated the m6A-LPS which contains five IncRNAs and coefficient of

each (Fig. 4a, d). The m6A-LPS involved five IncRNAs, and for each patient in the TCGA-Train, a risk score was calculated based on the coefficient for each IncRNA. Patients in the TCGA-Train were divided into low and high-risk subgroups based on the median value of risk scores. Kaplan-Meier survival curves depicted that BLCA patients with higher risk scores had worse clinical outcomes (lower OS rates and a shorter OS time) (Fig. 4b). Risk score and survival status distributions are plotted in Fig. 5A. And the ROC curves demonstrated that m6A-LPS harbored a promising ability to predict OS in the TCGA-Train (AUC = 0.713; Fig. 4e).

\section{Validation Of The M6a-Ips In The Tcga-test Dataset}

To validate the prognostic ability of m6A-LPS, we calculated risk scores for patients in the TCGA-Test cohort using the same formula. BLCA patients in the TCGA-Test dataset were assigned to low-risk and high-risk groups based on the median risk score. The results were consistent with the findings in the TCGA-train dataset: BLCA patients with higher risk scores had lower OS rates and a shorter OS time in the TCGA-test dataset (Fig. 4c). Risk score and survival status distributions showed that patients with higher risk scores had shorter OS time and dead status (Fig. 5c). The ROC analysis also indicated that m6A-LPS had a strong prognostic value for BLCA patients in the TCGA-test dataset (AUC $=0.682$; Fig. $4 f$ ). These results showed that the prognostic model of m6A-LPS had a robust and stable OS-predictive ability.

\section{The m6A-LPS was an independent prognostic factor for BLCA patients}

We used univariate and multivariate Cox analyses to assess whether the m6A-LPS was an independent prognostic factor for patients with BLCA. Based on the data of BLCA patients in the TCGA-train dataset, 
univariate Cox analysis indicated that the m6A-LPS was remarkably associated with OS [hazard ratio (HR):1.329,95\% Cl: 1.176-1.501, $\mathrm{p}<0.001$ ] and multivariate Cox analysis further showed that the prognostic model of m6A-related IncRNAs was an independent predictor of OS (HR: 1.302, 95\% Cl: 1.147-1.478, $p<0.001$; Fig. 5b). The conclusion was validated in the TCGA-test dataset, which confirmed that the prognostic model of m6A-related IncRNAs was an independent predictor of OS for BLCA patients in the TCGA-test validation dataset (univariate: HR: 3.027, 95\% Cl: 1.645-5.571, p < 0.001; multivariate: HR: $3.272,95 \%$ Cl: $1.601-6.685, p=0.001$; Fig. 5d). These results indicated that our the m6A-LPS, as an independent prognostic indicator, might be useful for clinical prognosis evaluation.

\section{Relationship between the m6A-LPS and multiple linicopathological features of BLCA}

The m6A-LPS vary differently among clinicopathological characteristics (Fig. 6a). When the data is divided into high-risk and low-risk groups, it can be observed that AL136295.2, AC104564.3, EHMT2 AS1 and AC116914.2 are in a high expression state in the low-risk group, while TP1B3 - AS1 were highly expressed in the high-risk group. Afterward, we tried to determine whether clinicopathological characteristics are related to m6A-LPS. Above all, we conducted a stratified analysis to confirm whether the risk score is different in clinical subgroups. In order to better show the correlation between clinicopathological characteristics and risk score, a sample with a higher BLCA risk score was deleted. The results showed that BLCA patients with stage III-IV, cluster 2, Age > 65 years, and T3-4 stage had a higher risk score. Low-Grade and ImmuneScore had a lower risk score (Fig. 6b-g). In addition, we conducted a stratified analysis to confirm whether it retains its ability to predict OS in each subgroup for better evaluate the prognostic ability of the risk Score. In contrast with patients with lower risk, higher risk BLCA patients had worse OS in the aged $\leq 65$ or $>65$ years, Male, Grade, T0-2 or T3-4 stage, M0 stage, N0-1, and Stage I-II or Stage III-IV subgroups (Fig. 6i-r). We also analyzed the vital immune checkpoints, i.e. The Kruskal-Wallis test was used to detect the significant differences between the Foxp3 expression level in two distinct cluster subtypes (Fig. 6h). From the above findings we concluded that the novel prognostic signature integrated by m6A-LPS could independently predict the prognosis of BLCA patients.

\section{Effect of risk score alterations of the m6A-LPS on immune cell infiltration}

The relationship between the risk score and infiltration levels of twenty-two immune cell types was analyzed to estimate the effect of five the m6A-related IncRNA prognostic signatures on the BLCA immune microenvironment. A significantly negative correlation was observed between the risk score and infiltration levels of the plasma cells, naive B cells, CD8 T cells, Tregs, and T cells gamma delta $(p<0.05$; Fig. 7a-e). The risk score was positively correlated with the infiltration levels of neutrophils, mast cells activated,NK cells resting, M0 macrophages and M1 macrophages $(p<0.05 ;$ Fig. $7 f-j)$. This result confirmed that $\mathrm{m6A}$ regulator-based risk signatures were implicated in the BLCA immune microenvironment.

\section{Discussion}


m6A mRNA modifications are evolving to be a new layer of posttranscriptional gene regulation. The dynamic and delicate interplay between m6A methyltransferases and demethylases plays crucial roles in many fundamental biological processes, such as RNA splicing, protein translation and cell fate transition[20]. IncRNA markers, often used to predict prognosis. But m6A-related IncRNAs are rarely used to predict the prognosis of BLCA. We screened IncRNAs related to prognosis from 714 immune-related IncRNAs. Using univariate Cox proportional hazards regression analysis, we finally obtained nine prognostic m6A-related IncRNAs. Nine prognostic m6A-related IncRNAs were significantly different between tumor and non tumor tissues of BLCA, and cluster analysis showed that they had the potential to predict the prognosis of bladder cancer. Using Lasso regression analysis and multivariate Cox proportional hazards regression analysis, we finally obtained 5 independent prognostic m6A-related IncRNAs. Consequently, we constructed a m6A-related IncRNAs prognostic signature (m6A-LPS,including AL136295.2, AC104564.3, ATP1B3-AS1, EHMT2-AS1 and AC116914.2) based on the ability of 5 m6Arelated IncRNAs to predict the OS of BLCA patients. Survival analysis, clinical correlation analysis, independent prognostic analysis and correlation analysis of immune cell infiltration were performed on these IncRNAs. The results show that m6A-LPS is scientific and reasonable. The role of AL136295.2, AC104564.3, ATP1B3-AS1, EHMT2-AS1 and AC116914.2 in BLCA has not been reported. These m6Arelated IncRNAs may be new markers for predicting the prognosis of BLCA, as well as new targets for immunotherapy, but further basic and clinical laboratory identification are needed. All the evidence shows that m6A modification is targeted at IncRNAs, therefore we need to pay more attention to the interactions and functions of IncRNAs and m6A modifications so as to identify potential prognostic markers or therapeutic targets of BLCA.

In this study, BLCA was divided into two different subtypes. We found that cluster 1 has a high penetration of Tregs. Treg cells are usually enriched in tumor tissues. The high rate of tumor infiltrating Treg cells (TIL Tregs) usually indicates adverse clinical outcomes in bladder cancer [21].Our study found that compared with cluster 2, the expression of m6A related IncRNAs in cluster 1 was higher and the prognosis was better. The results showed that the methylation density of cluster 1 was higher than that of cluster2. It is suggested that the higher the density of Tregs methylation in BLCA tissue, the better the prognosis of patients. However, both lymphoid Treg cells and tissue resident Treg cells need to express Foxp3 transcription factor. Foxp3 protein functions in transcriptional molecule complexes. Ensemble formation is regulated by various interactions and post-translational modifications (PTMs), including phosphorylation, O-GIcNAcylation, acetylation, ubiquitination, and methylation [22]. Modifications influence each other to orchestrate Foxp3 activity and Treg suppression, indicating that Foxp3 is essential for Treg function. Most notably, the stable expression of Foxp3 can only be found in Treg cells with enhanced The Treg-specific demethylation region (TSDR) demethylation [23]. Gene analysis showed that Foxp3 was significantly different between the two subtypes, and the invasion density of Foxp3 was higher in bladder cancer. It is suggested that the inhibition of Treg by methylation of Foxp3 gene is the reason for better prognosis of bladder cancer. According to the above results, understanding the inhibition of Foxp3 + Treg methylation may help to design a reasonable immunotherapy regimen for bladder cancer. 
We observed that there were relatively high estimated score in cluster 2 . The higher the estimated score, the higher the purity of tumor. The high infiltration rate of immunoscore was positively correlated with the increased infiltration of M2 macrophages, memory activated CD4 + T cells and neutrophils. However, memory activated CD4 + T cells, Tumor-associated M2 macrophages and neutrophils infiltration increased, which was described as "Immune failure related phenotypes" by previous studies [24, 25]. In addition, tumor associated M2 macrophages and inflammation associated neutrophils can express cytokines and enzymes that inhibit T cell recruitment and activation [26, 27]. Previous studies found that compared with patients with negative Foxp3 expression in BLCA cells, increased Foxp3 expression suggests shorter survival time [28]. The high count of Tumor-associated M2 macrophages was associated with shorter relapse free survival [29]. Consistent with our study, Foxp3 is highly expressed in bladder cancer and has poor prognosis. These results also suggest that Foxp3 demethylation may also affect the invasion of Tumor-associated M2 macrophages and increase the risk of bladder cancer. KEGG enrichment analysis further confirmed our study.

This study has certain limitations. First of all, the construction of m6A-LPS only sets up the internal validation set but not the external validation set. Therefore, this study did not evaluate the influence of external effects on the prognostic model. Secondly, Foxp3 + Treg methylation and Foxp3 + Tumorassociated M2 macrophages demethylation may be new targets for immunotherapy, and further basic and clinical laboratory identification are needed.

\section{Conclusions}

We extracted the expression matrixes of $21 \mathrm{m6A}$-related genes from the TCGA datasets, respectively. Pearson correlation analysis was performed to search 741 m6A-related IncRNAs. univariate Cox regression was performed to screen nine the prognosis of from 741 IncRNAs related to m6A. LASSO Cox analysis on the basis of the 9 m6A-related prognostic IncRNAs and it generated the m6A-LPS (Including AL136295.2ロAC104564.3ロATP1B3-AS1EHMT2-AS1 and AC116914.2). This signature was capable to predict the OS of Patients in different cohort and served as an independent prognostic factor for BLCA. In the consensus cluster analysis, we found that the higher the infiltration rate of Tregs in the context of methylation, the better the prognosis of bladder cancer patients. Gene analysis showed that Foxp3 methylation may lead to the inhibition of Tregs function, which is one of the reasons for the better prognosis of bladder cancer. In addition, Foxp3 demethylation may also affect the invasion of M2 macrophages and increase the risk of bladder cancer. In conclusion, m6A related IncRNAs may be new markers for predicting the prognosis of BLCA, and Foxp3 + Tregs methylation and Foxp3 + Tumorassociated M2 macrophases demethylation may be new targets for immunotherapy of bladder cancer.

\section{Abbreviations}

BLCA : Bladder cancer; m6A-related IncRNAs; N6-methylandenosine-related long non-coding RNAs;m6ALPS: m6A-related IncRNA prognostic signature; GENCODE: Encyclopædia of genes and gene variants; CIBERSORT: cell type abundances from bulk tissue transcriptomes; ESTIMATE: Estimation of 
StromalScore and Immune cells in malignant Tumor tissues using Expression data; LASSO: least absolute shrinkage and selection operator; TCGA: The Cancer Genome Atlas; KEGG: Kyoto Encyclopedia of Genes and Gene Ontology; GO: Gene ontology; K-M: Kaplan-Meier; ROC: Receiver operating characteristic; OS: Overall survival;

\section{Declarations}

\section{Acknowledgements}

Not applicable.

\section{Authors' contributions}

$\mathrm{HHZ}$, PPX and YDL designed the study. HHZ and KJS collected the mRNA transcriptome data and clinical information from TCGA. HHZ, CCY and JZZ performed analyses on TCGA data. XRC HHZ and DNX performed statistical analyses. HHZ wrote the manuscript. HHZ, PPX, KJS, XRCQ DNX, JZZ, CCY and YDL reviewed and revised the manuscript. All authors read and approved the final manuscript.

\section{Funding}

The work was supported by the Key Research and Development Project of Hainan Province (ZDYF2019148), and the National Natural Science Foundation of China (No.81960549).

\section{Availability of data and materials}

Data and materials are available for sharing if needed

Ethics approval and consent to participant

Not applicable.

\section{Consent for publication}

Not applicable.

\section{Competing interests}

The authors declare that there are no conflicts of interest.

\section{Author details}

1 Department of Medical Oncology, The First Affiliated Hospital of Hainan Medical College, Hainan Medical College, Haikou, Hainan $₫ 570000$, P.R. China;

2 Hainan Provincial Key Laboratory for Tropical Cardiovascular Diseases Research, the First Affiliated Hospital of Hainan Medical University, Haikou, Hainan囚571199, P.R. China. 
3 Department of Medical Oncology and Tumor Institute, The First Affiliated Hospital of Hainan Medical University, Haikou, Hainan $₫ 570102$, P.R. China.

4 Key Laboratory of Emergency and Trauma of Ministry of Education, Research Unit of Island Emergency Medicine of Chinese Academy of Medical Sciences, Key Laboratory of Tropical Cardiovascular Diseases Research of Hainan Province, Hainan Medical University, Haikou, Hainan $₫ 571199$, P.R. China.

\section{References}

1. Song J, Xu Q, Zhang $\mathrm{H}$, et al. Five key IncRNAs considered as prognostic targets for predicting pancreatic ductal adenocarcinoma. J Cell Biochem. 2018;119(6):4559-69.

2. Lossos IS, Czerwinski DK, Alizadeh AA, et al. Prediction of survival in diffuse large-B-cell lymphoma based on the expression of six genes. N Engl J Med. 2004;350(18):1828-37.

3. Yu G, Wang LG, Han Y, He QY. clusterProfiler: an R package for comparing biological themes among gene clusters. OMICS. 2012;16(5):284-7.

4. Gentles AJ, Newman AM, Liu CL, et al. The prognostic landscape of genes and infiltrating immune cells across human cancers. Nat Med. 2015;21(8):938-45.

5. Yoshihara K, Shahmoradgoli M, Martínez E, et al. Inferring tumour purity and stromal and immune cell admixture from expression data. Nat Commun. 2013;4:2612.

6. Friedman J, Hastie T, Tibshirani R. Regularization Paths for Generalized Linear Models via Coordinate Descent. J Stat Softw. 2010;33(1):1-22.

7. Tao C, Huang K, Shi J, Hu Q, Li K, Zhu X. Genomics and Prognosis Analysis of EpithelialMesenchymal Transition in Glioma. Front Oncol. 2020;10:183.

8. Ritchie ME, Phipson B, Wu D, et al. limma powers differential expression analyses for RNAsequencing and microarray studies. Nucleic Acids Res. 2015;43(7):e47.

9. Blanche P, Dartigues JF, Jacqmin-Gadda $\mathrm{H}$. Estimating and comparing time-dependent areas under receiver operating characteristic curves for censored event times with competing risks. Stat Med. 2013;32(30):5381-97.

10. Bray F, Ferlay J, Soerjomataram I, Siegel RL, Torre LA, Jemal A. Global cancer statistics 2018 : GLOBOCAN estimates of incidence and mortality worldwide for 36 cancers in 185 countries. CA Cancer J Clin. 2018;68(6):394-424.

11. Alfred Witjes J, Lebret T, Compérat EM, et al. Updated 2016 EAU Guidelines on Muscle-invasive and Metastatic Bladder Cancer. Eur Urol. 2017;71(3):462-75.

12. Cambier S, Sylvester RJ, Collette L, et al. EORTC Nomograms and Risk Groups for Predicting Recurrence, Progression, and Disease-specific and Overall Survival in Non-Muscle-invasive Stage TaT1 Urothelial Bladder Cancer Patients Treated with 1-3 Years of Maintenance Bacillus CalmetteGuérin. Eur Urol. 2016;69(1):60-9. 
13. Szabados B, van Dijk N, Tang YZ, et al. Response Rate to Chemotherapy After Immune Checkpoint Inhibition in Metastatic Urothelial Cancer. Eur Urol. 2018;73(2):149-52.

14. Zhao BS, Roundtree IA, He C. Post-transcriptional gene regulation by mRNA modifications. Nat Rev Mol Cell Biol. 2017;18(1):31-42.

15. Zaccara S, Ries RJ, Jaffrey SR. Reading, writing and erasing mRNA methylation. Nat Rev Mol Cell Biol. 2019;20(10):608-24.

16. Ma S, Chen C, Ji X, et al. The interplay between m6A RNA methylation and noncoding RNA in cancer. J Hematol Oncol. 2019;12(1):121.

17. Patil DP, Chen CK, Pickering BF, et al. m(6)A RNA methylation promotes XIST-mediated transcriptional repression. Nature. 2016;537(7620):369-73.

18. Han J, Wang JZ, Yang X, et al. METTL3 promote tumor proliferation of bladder cancer by accelerating pri-miR221/222 maturation in m6A-dependent manner. Mol Cancer. 2019;18(1):110.

19. Horn T, Laus J, Seitz AK, et al. The prognostic effect of tumour-infiltrating lymphocytic subpopulations in bladder cancer. World J Urol. 2016;34(2):181-7.

20. Liu GM, Zeng HD, Zhang CY, Xu JW. Identification of METTL3 as an Adverse Prognostic Biomarker in Hepatocellular Carcinoma. Dig Dis Sci. 2021;66(4):1110-26.

21. Pichler R, Fritz J, Zavadil C, Schäfer G, Culig Z, Brunner A. Tumor-infiltrating immune cell subpopulations influence the oncologic outcome after intravesical Bacillus Calmette-Guérin therapy in bladder cancer. Oncotarget. 2016;7:39916-30.

22. Deng G, Song X, Fujimoto S, Piccirillo CA, Nagai Y, Greene MI. Foxp3 Post-translational Modifications and Treg Suppressive Activity. Front Immunol. 2019;10:2486.

23. Polansky JK, Kretschmer K, Freyer J, et al. DNA methylation controls Foxp3 gene expression. Eur J Immunol. 2008;38(6):1654-63.

24. Chen YP, Wang YQ, Lv JW, et al. Identification and validation of novel microenvironment-based immune molecular subgroups of head and neck squamous cell carcinoma: implications for immunotherapy. Ann Oncol. 2019;30(1):68-75.

25. Biswas SK, Mantovani A. (2010). Macrophage plasticity and interaction with.

26. Iymphocyte subsets. cancer as a paradigm. Nat. Immunol. 11, 889-896.

27. Cai T, Nesi G, Boddi V, Mazzoli S, Dal Canto M, Bartoletti R. Prognostic role of the tumor-associated tissue inflammatory reaction in transitional bladder cell carcinoma. Oncol Rep. 2006;16(2):329-34.

28. Jung KY, Cho SW, Kim YA, et al. Cancers with Higher Density of Tumor-Associated Macrophages Were Associated with Poor Survival Rates. J Pathol Transl Med. 2015;49(4):318-24.

29. Winerdal ME, Marits $P$, Winerdal M, et al. FOXP3 and survival in urinary bladder cancer. BJU Int. 2011;108(10):1672-8.

30. Miyake M, Tatsumi Y, Gotoh D, et al. Regulatory T Cells and Tumor-Associated Macrophages in the Tumor Microenvironment in Non-Muscle Invasive Bladder Cancer Treated with Intravesical Bacille 
Calmette-Guérin: A Long-Term Follow-Up Study of a Japanese Cohort. Int J Mol Sci. 2017;18(10):2186.

\section{Figures}

a $\quad$ IncRNA $\bullet$ m6A b
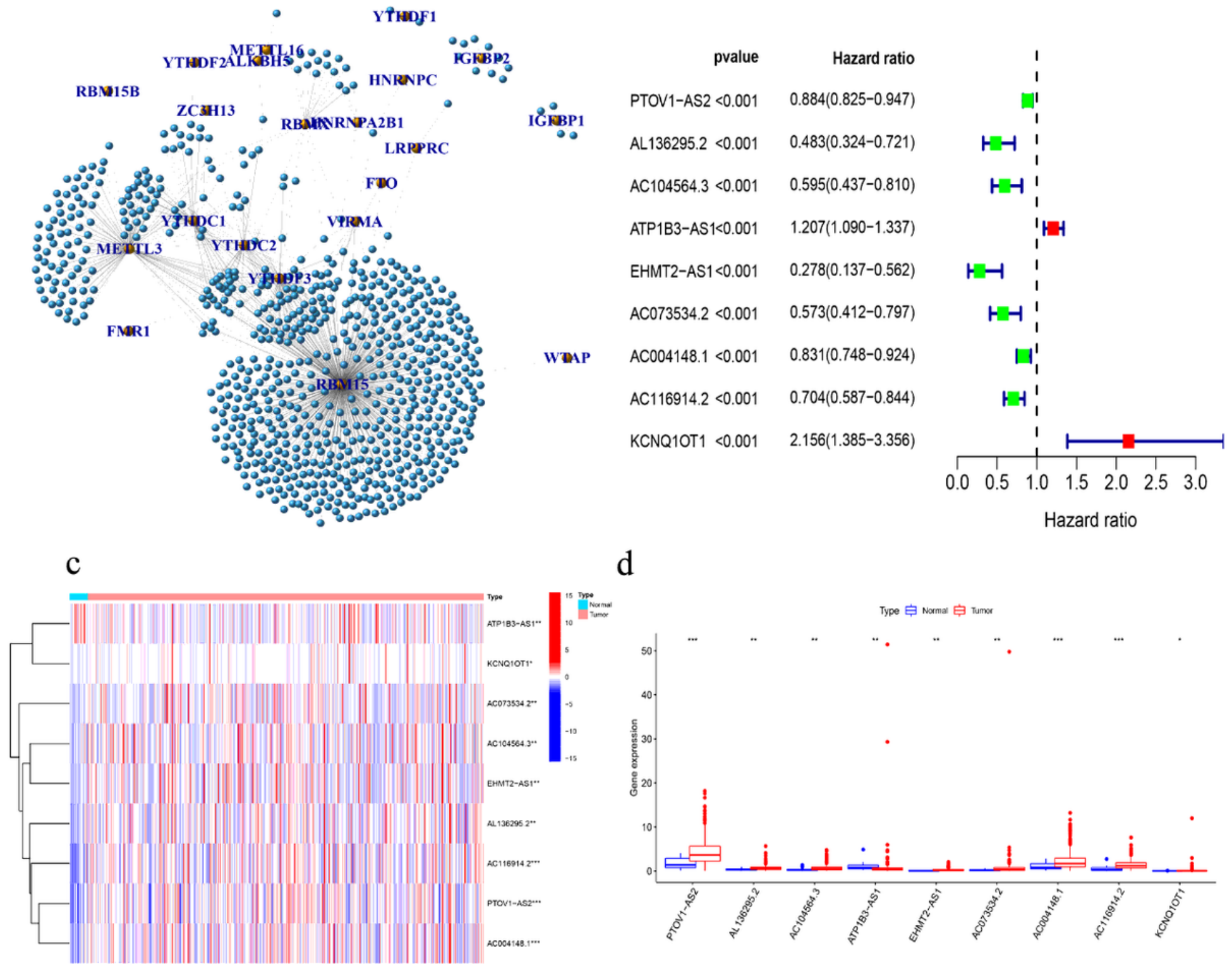

d

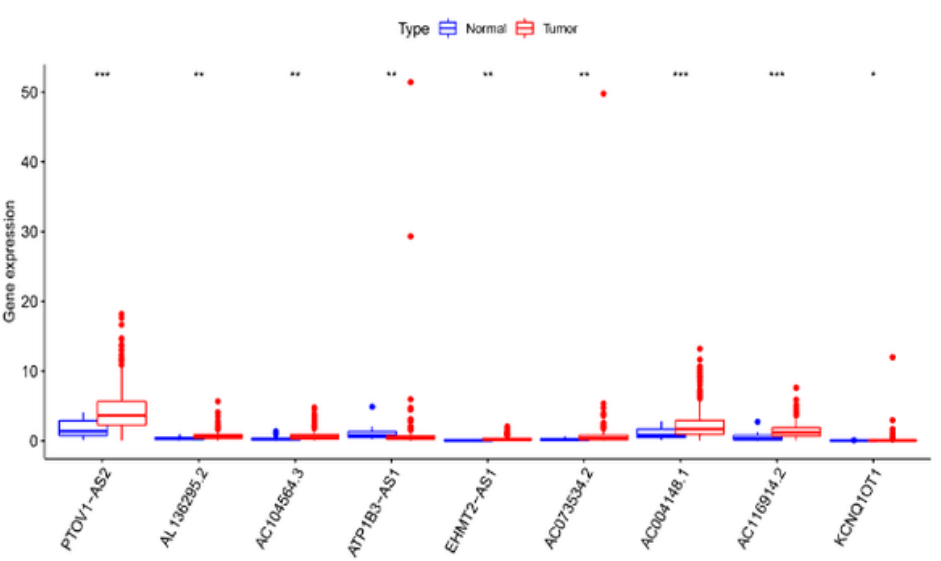

Figure 1

Transcriptome profiles of the m6A-related IncRNAs in BLCA. a m6A-related genes and IncRNA coexpression network of BLCA. b Forest plot of univariate Cox regression analysis for the m6A-related IncRNAs. c, d The differential expression of the m6A-related IncRNAs between 407 tumor tissues and 19 normal tissues. 
Fig.2

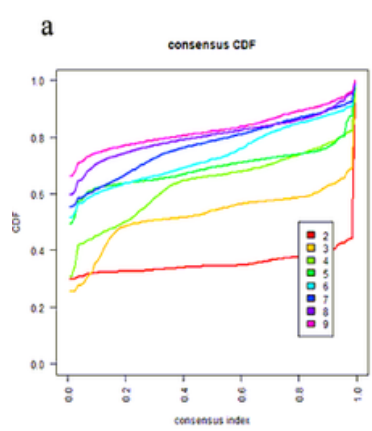

f

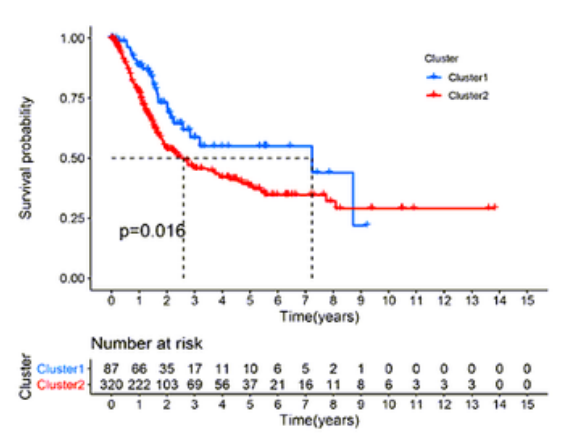

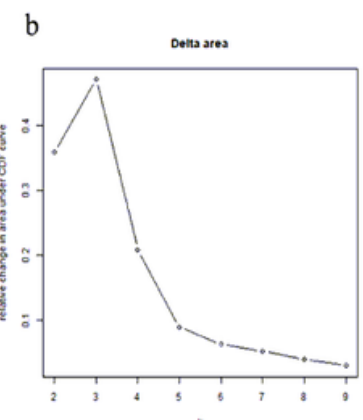

g
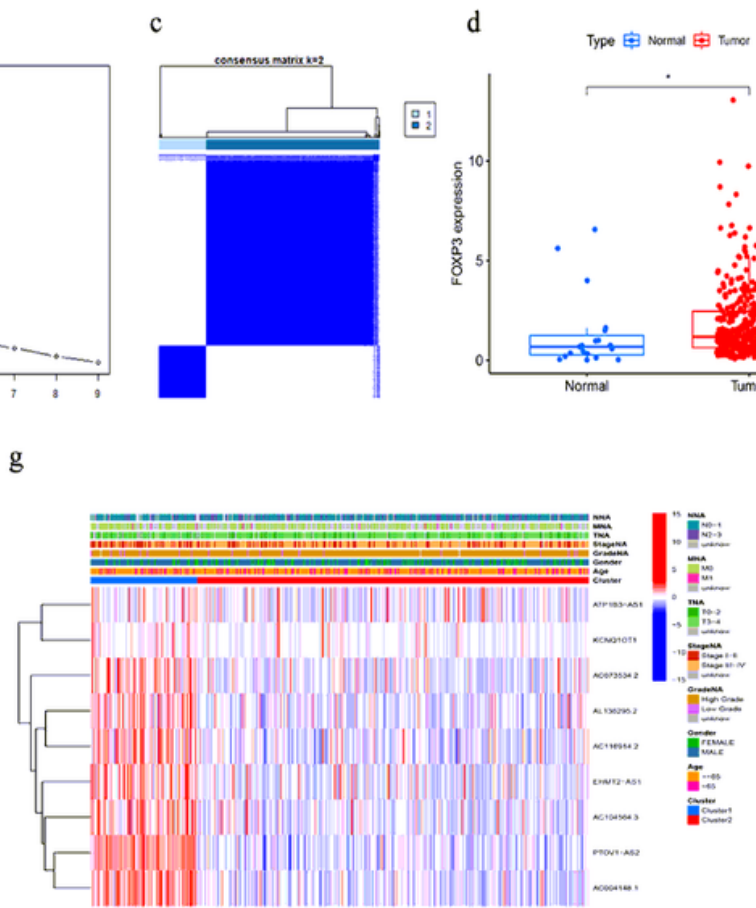
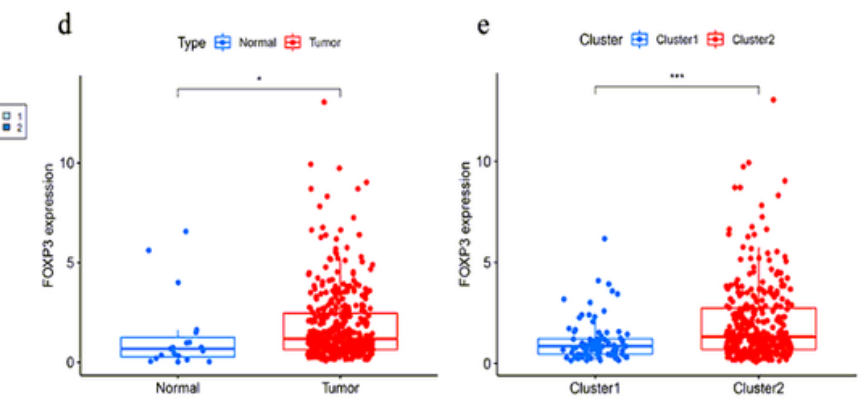

$\mathrm{h}$

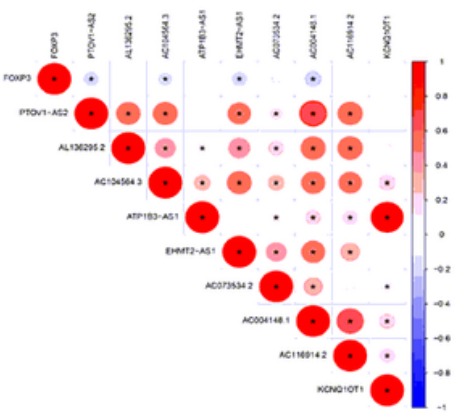

Figure 2

Association between the m6A-related IncRNAs and clinicopathological and prognostic features of BLCA patients. a Consensus clustering model with CDF for $k=2-9$. $b$ Relative change in area under the CDF curve for $\mathrm{k}=2-9$. c TCGA-BLCA cohort was classified into two clusters with $\mathrm{k}=2$. $\mathrm{d}$ Foxp3 expression clusters levels. e Expression of Foxp3 in BLCA tissue and normal bladder tissue. f Kaplan-Meier OS curves for patients in distinct clusters $(p=0.016)$. $g$ The heatmap of nine constituent IncRNAs of risk signature along with clinicopathological characteristics. $\mathrm{h}$ Correlations between the nine m6A-related IncRNAs and Foxp3. 

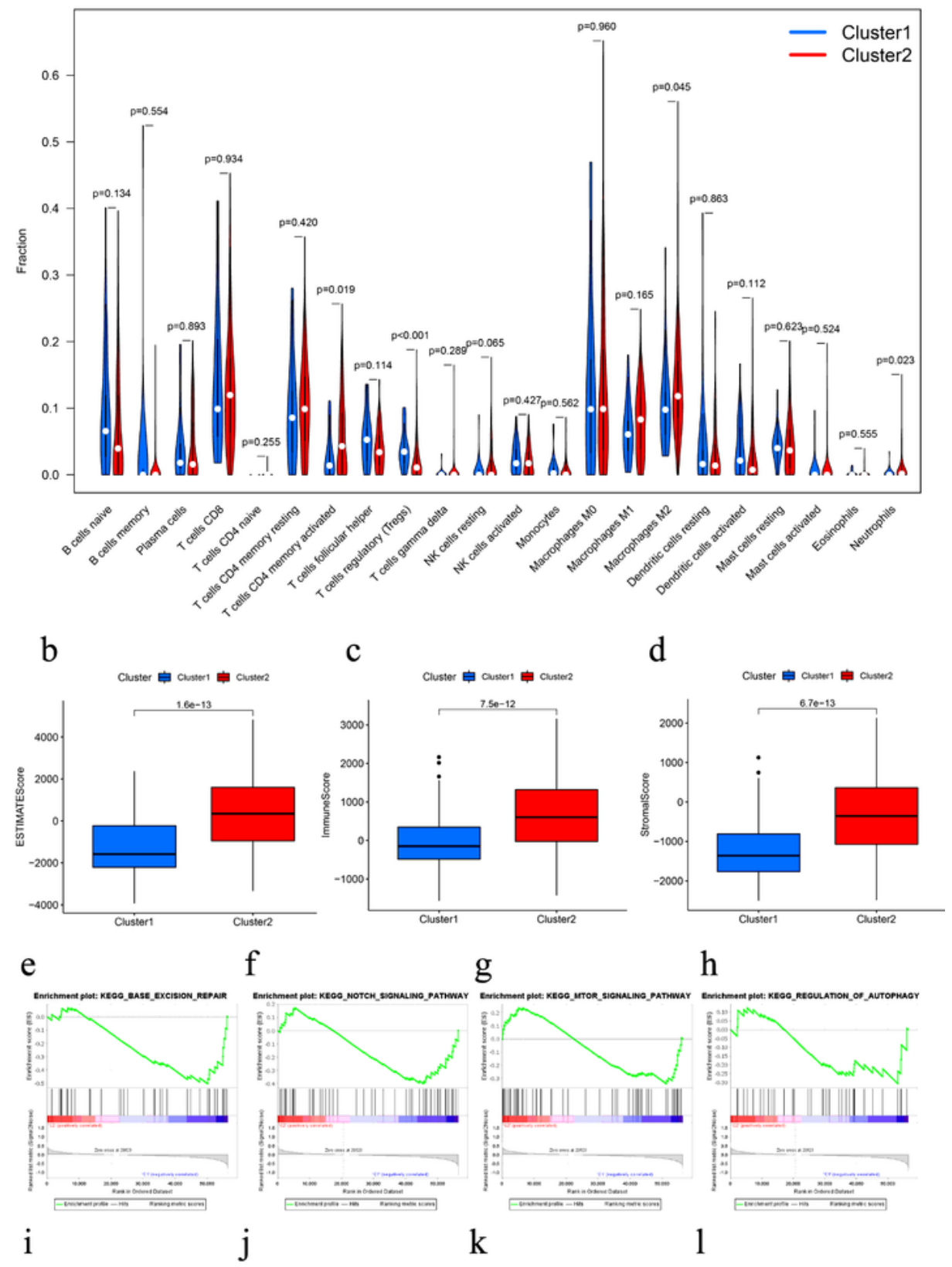

$\mathrm{h}$
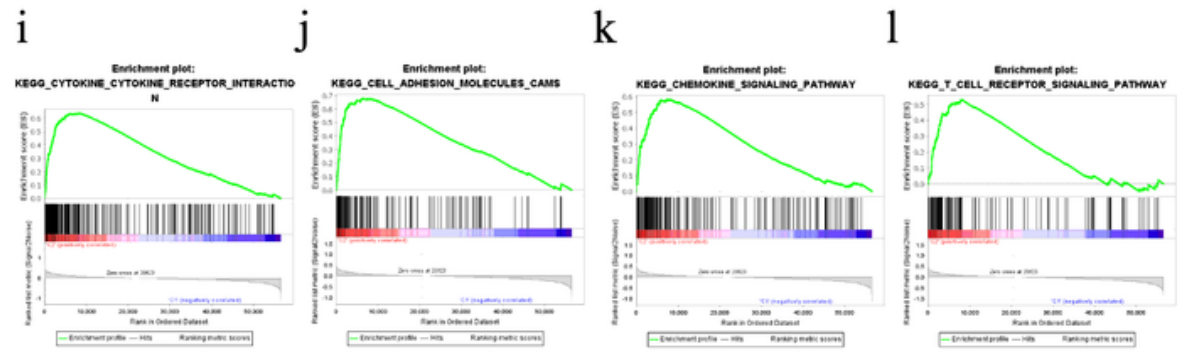

\section{Figure 3}

Consensus clustering for m6A-related IncRNAs associated with distinct immune cell infiltration. a Cellular interaction of the tumor-infiltrating immune cell types. b-d immune microenvironment difference in the m6A-related IncRNAs subtypes. (Wilcoxon test, $p$ <0.05). e-I GSEA enrichment analysis is in the cluster 1 (e-h) or cluster $2(\mathrm{i}-\mathrm{I})$ subgroup. 
a

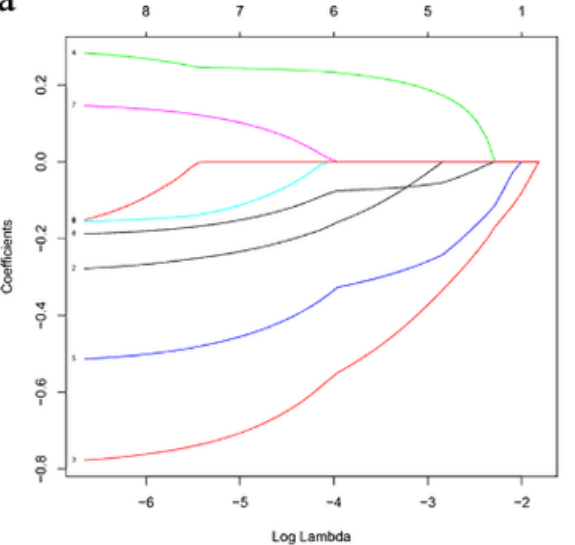

d

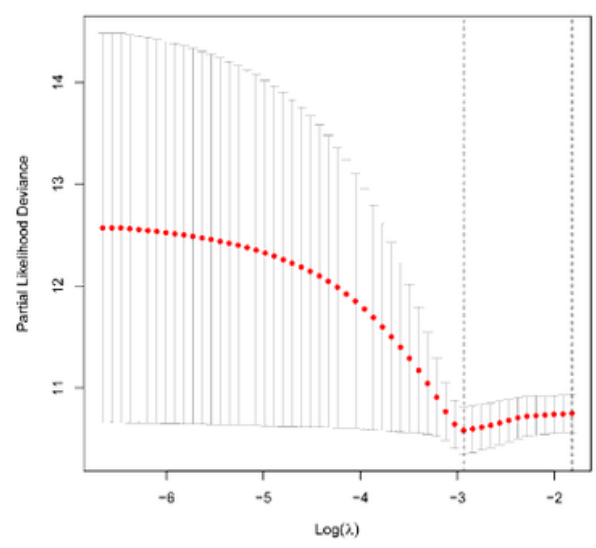

b

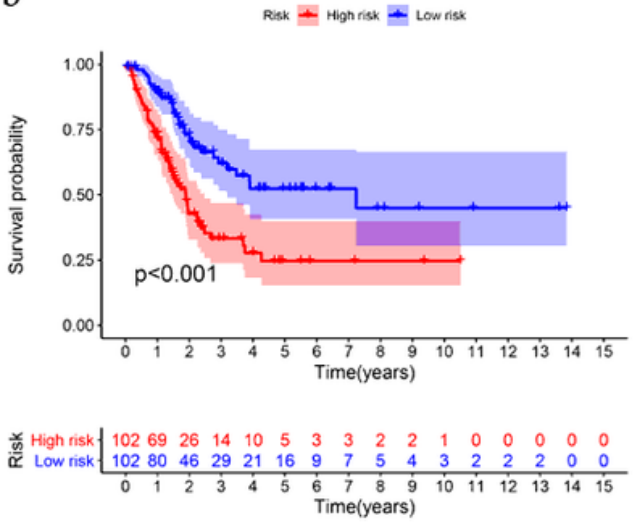

$\mathrm{e}$

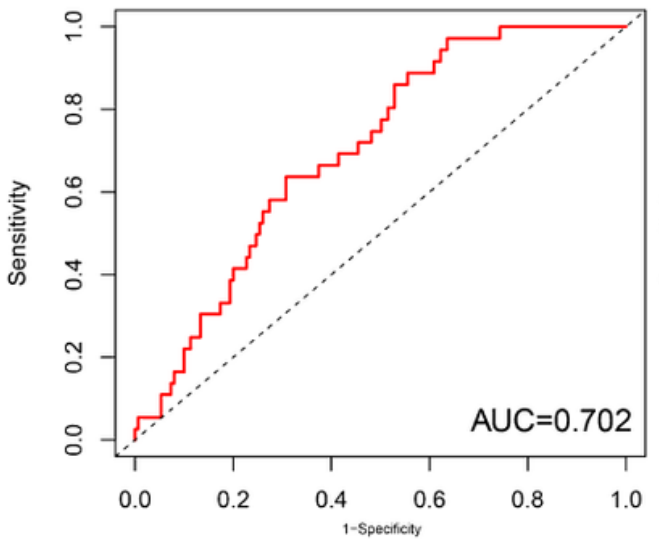

c

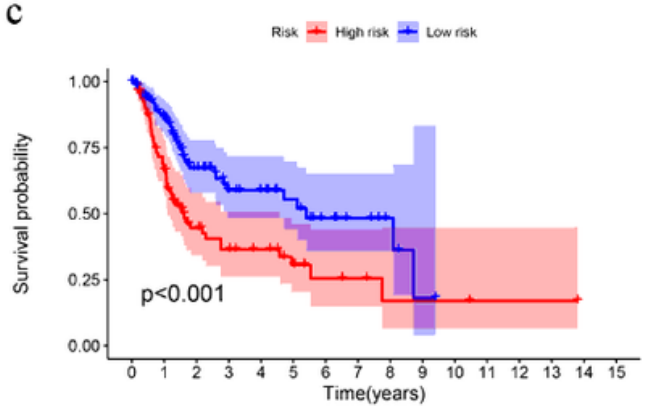

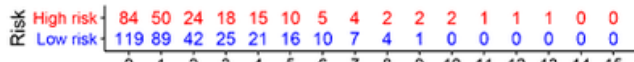
$\begin{array}{ccc}6 & 7 & 8 \\ \text { Time(years) }\end{array}$

f

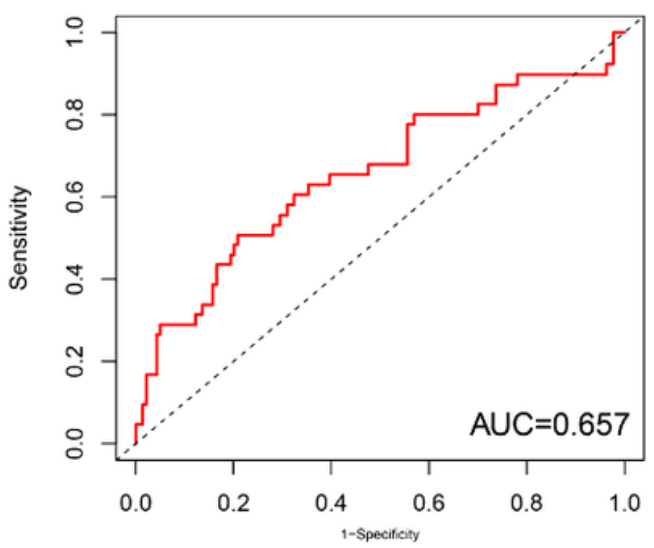

Figure 4

Identification of five critical m6A-related IncRNAs. a, d LASSO regression was performed, calculating the minimum criteria. b Kaplan-Meier curves showed that the high-risk subgroup had worse overall survival than the low-risk subgroup in TCGA-train dataset. c Kaplan-Meier curves showing that the high-risk subgroup had worse overall survival than the low-risk subgroup in TCGA-test dataset. e, $f$ ROC curve analysis on the accuracy of m6A-LPS in BLCA to predict the prognosis of patients. 

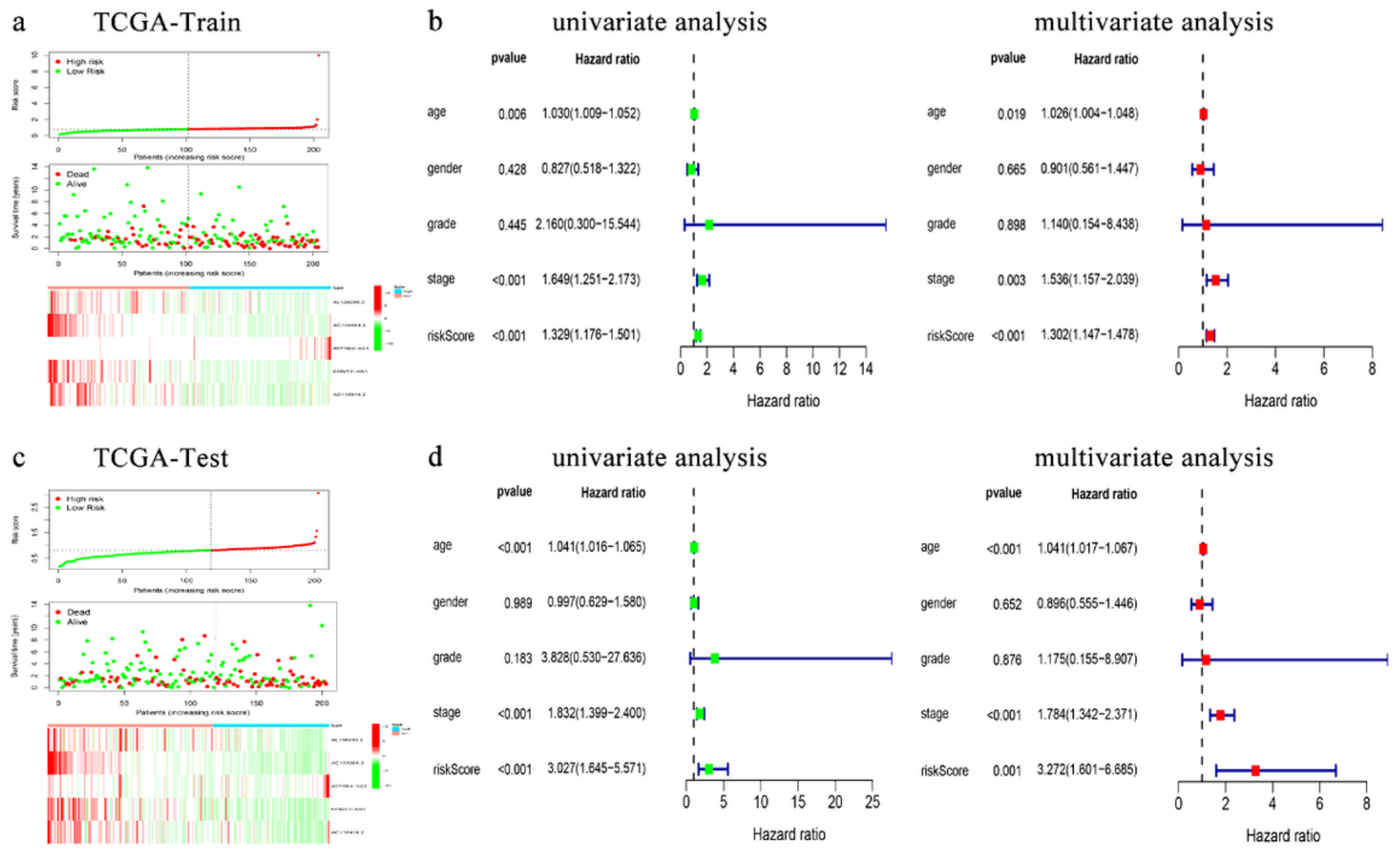

Figure 5

The efficacy of novel risk signature consisted of five m6A-related IncRNAs. a Distribution of risk scores [based on the m6A-LPS] and survival status of BLCA patients in the TCGA-train dataset. c Distribution of risk scores and survival status of BLCA patients in the TCGA-Test dataset. b, $d$ Univariate and multivariate analysis revealed that m6A-LPS was an independent prognostic predictor in the TCGA-train and TCGAtest datasets. 

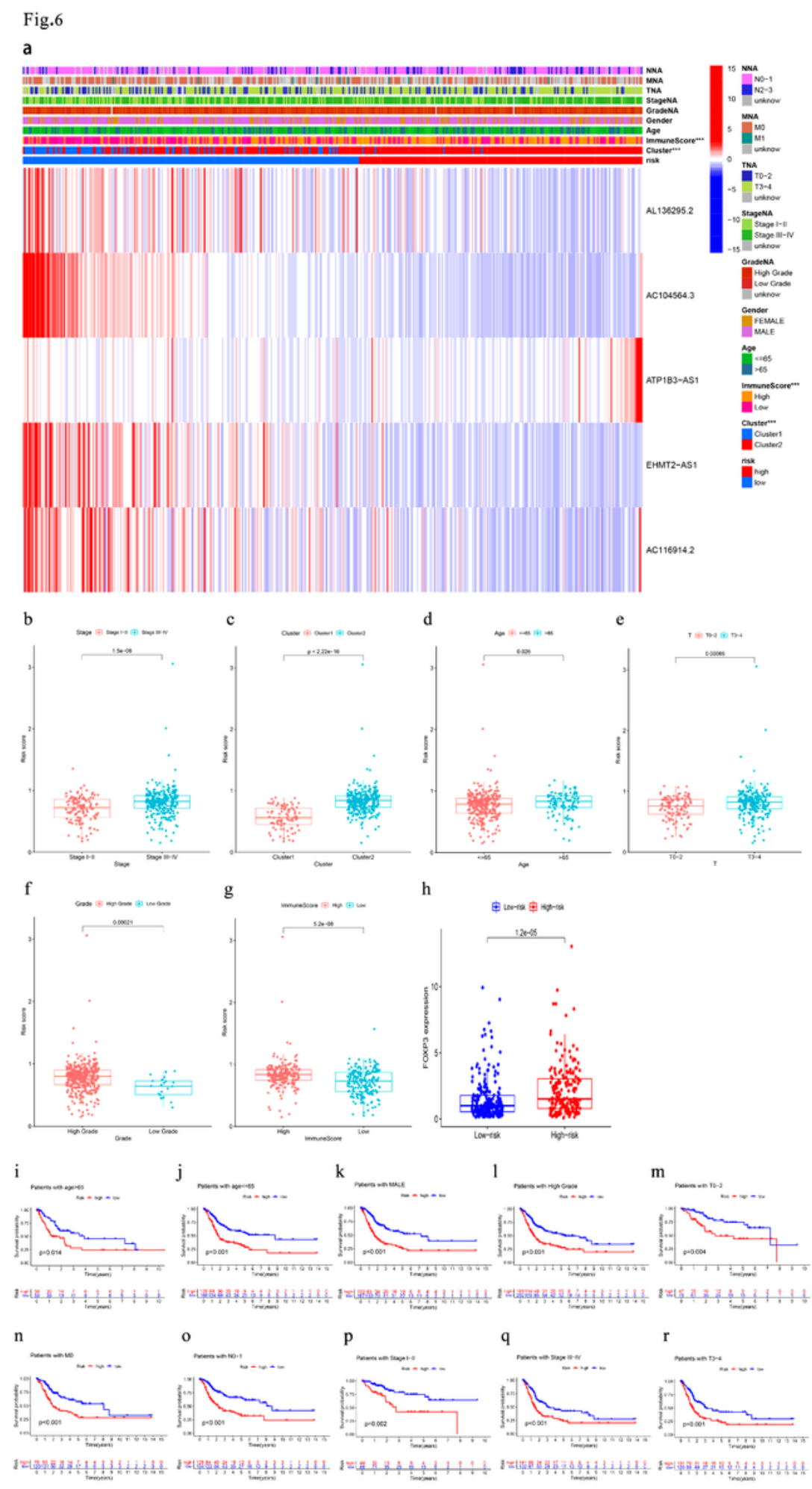

\section{Figure 6}

Clinicopathological characteristics of novel risk signature constituted of the m6A-LPS. a The heatmap of five constituent IncRNA of risk signature along with clinicopathological characteristics. b-g Patients with different clinicopathological features (including stage III-IV, cluster 2, Age> 65 years, T3-4 stage, LowGrade, ImmuneScore) had different levels of risk scores, calculated based on the m6A-LPS. h Foxp3 expression among distinct clusters (Kruskal-Wallis test, $p<0.001)$. $i-r$ The constituent prognostic model 
of m6A-LPS retained its prognostic value in multiple subgroups of BLCA patients (including patients with aged $\leq 65$ or $>65$ years, Male, Grade, T0-2 or T3-4 stage, M0 stage, N0-1 and patients with stage I-II or stage III-IV ).

Fig.7
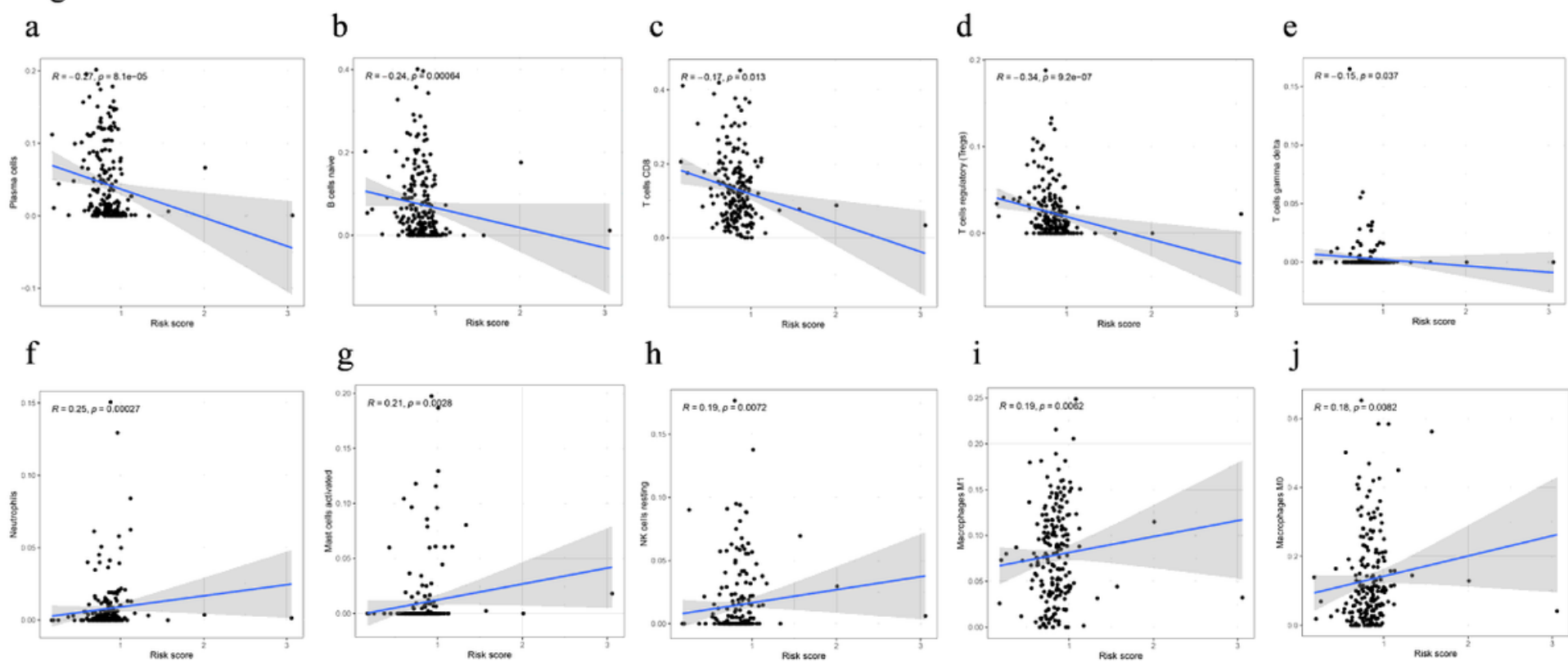

$\mathrm{j}$
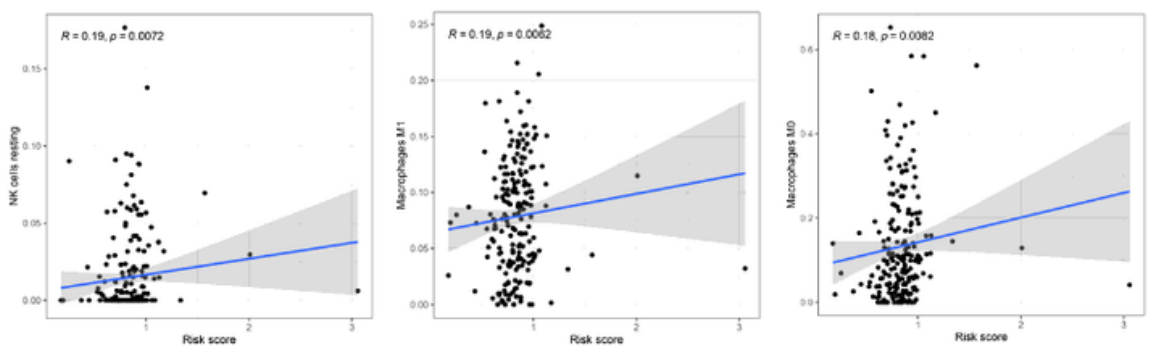

\section{Figure 7}

Relationships between the risk score and infiltration abundances of ten immune cell types. a-e Plasma cells (a), naive B cells (b), CD8 T cells (c), Tregs (d), T cells gamma delta(e). f-j Neutrophils (f), Mast cells activated (g), NK cells resting (h), M0 macrophages(i), and M1 macrophages (j). 